\section{Chance findings}

Think of mRNA splicing and the first image that comes to mind is the process of snipping out the non-coding segments from a pre-mRNA, allowing exons to be strung together into a mature message. Indeed, cis-splicing events occur in most mRNAs in vertebrate cells. But not all exons derive from their pre-mRNA — in many organisms, exons from different RNA molecules fuse to form a mature message, in a process called trans-splicing. A rare form of trans-splicing, called spliced leader (SL) trans-splicing, has been reported only in several protists and in less-advanced animal lineages. Now, Vandenberghe et al. provide evidence of SL trans-splicing in a primitive chordate, Ciona intestinalis, raising the possibility that this process also occurs in vertebrates.

Trans-splicing isn't an easy phenomenon to spot, and most stumble on it by accident. This study is no exception. The researchers' original intention was to isolate the gene for the muscle protein troponin I (TnI) in the ascidian C. intestinalis. In doing so, they found that the first 16 nucleotides of the TnI mRNA were nearly identical in sequence to those of at least six other, functionally unrelated mRNAs.

Could the 16-nucleotide stretch be a common exon (the spliced leader) that is spliced onto the $5^{\prime}$ end of distinct acceptor RNAs? If so, then this would be the first example of SL trans-splicing in a chordate. That this was the case was confirmed by the absence of the 16 bases from the TnI genomic sequence and by the trans-splicing of the 16 nucleotides onto an artificial mRNA substrate that retained the $T n I$ splice acceptor site. The authors also identified a strong candidate for the splice donor molecule - a 46-nucleotide RNA species — from which the 16 bases derive.

This discovery of SL trans-splicing in chordates significantly extends the phylogenetic range in which the process is known to occur. Although opinions are divided as to the advantages of trans-splicing, knowing its phylogenetic distribution provides clues about how transand cis-splicing have evolved. These two processes might be closely related, as shown by the use of the same consensus splice acceptor/donor sites and the similarity between the SL RNA and a spliceosomal snRNA. But does SL trans-splicing occur in vertebrates? The answer might require some more serendipity.

Tanita Casci

\section{(2) References and links}

ORIGINAL RESEARCH PAPER Vandenberghe, A. E. et al. mRNA 5'-leader trans-splicing in the chordates. Genes Dev. 15, 294-303 (2001)

ENCYCLOPEDIA OF LIFE SCIENCES Urochordata

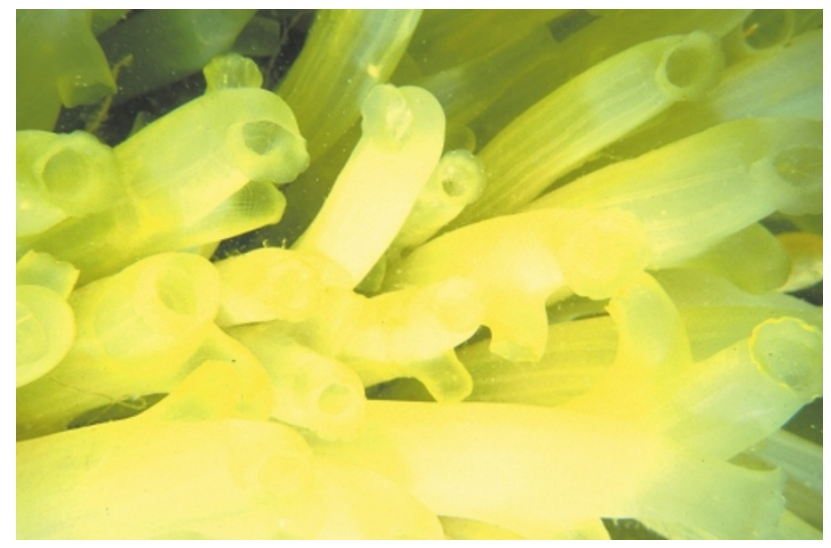

Courtesy of Sue Strome.

\section{Snapshots of metabolic phenotypes}

If you've recently knocked out a gene but your mutant has no obvious phenotype, then read on. Because a recent paper in Nature Biotechnology reports a new way to overcome the problem that many functional genomicists face - elucidating the function of a gene that produces no overt phenotype when inactivated.

To find phenotypes for such genes, Raamsdonk et al. delved into the Saccharomyces cerevisiae metabolome - its complement of $\sim 600$ cellular low molecular weight, metabolic intermediates.

Metabolites, like proteins, are functional properties of a cell and change according to a cell's physiological, developmental or pathological state. Because of this, the authors reasoned that the effects of some genetic mutations might be counteracted by metabolic fluxes that prevent overt phenotypes, such as altered growth rates, from occurring.

But there is a problem with this approach. How do you go from metabolic profile to gene function if there is often no direct relationship between the two? To overcome this obstacle, the authors developed a new approach called FANCY.

FANCY (for functional analysis by co-responses in yeast) is based on the measurement of the steady-state response of two variables to the change of a system parameter - that is, in this case, the change in concentrations of two metabolites in response to a mutation. The premise for this approach is that these responses should be similar in cells that have had the same functional response or pathway disrupted. In this way, matching the metabolic response to the loss of an unknown gene to that of a known gene should give clues as to the gene's function. The authors validated this approach by looking at two deletion mutants in the glycolysis metabolic pathway.

The two mutants had no growth-rate

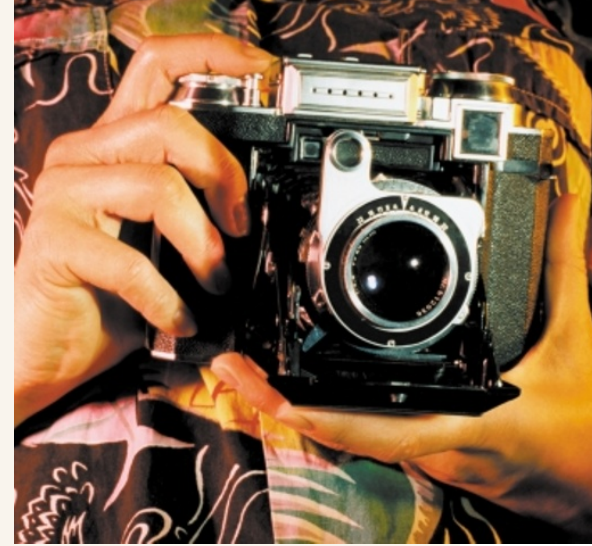

phenotype but did have a metabolic phenotype when the concentrations of six metabolites were measured.

Changes in the levels of these metabolites were similar in the glycolysis-deficient mutants, but were different to the changes in two respiratory-deficient mutants.

However, this approach is somewhat limited by the need to know which metabolites to measure. How could you predict this for genes of unknown function? So, to overcome this limitation, the authors went one step further and used nuclear magnetic resonance (NMR) spectroscopy to take 'metabolic snapshots' of cells. This approach can be used without knowing which metabolites a mutation affects. And Raamsdonk et al.'s stastistical analyses of the resulting NMR spectra could distinguish mutants with different metabolic phenotypes, and cause those with similar phenotypes to cluster together. These clusters can then be analysed by using known functional mutants to guide the analysis of co-clustering, poorly understood mutants.

This approach is now being used in a high-throughput screen of a library of $\sim 6000$ S.cerevisiae mutants, each of which has a single open reading frame removed from its genome. Because this approach can reveal the function of non-metabolic genes genes that are involved in protein biosynthesis, for example, the deletion of which would cause the cellular concentrations of certain amino acids to change - it promises to add to our growing knowledge of gene function, both in yeast and in higher organisms.

Jane Alfred

(9) References and links ORIGINAL RESEARCH PAPER Raamsdonk, L. M. et al. A functional genomics strategy that uses metabolome data to reveal the phenotype of silent mutations. Nature Biotechnol. 19, 45-50 (2001)

WEB SITES Stephen Oliver's lab | Douglas Kell's quantitative biology and analytical biotechnology group 